\title{
An improved algorithm for multiple source direction of arrival estimation
}

\author{
Xuecheng Wang ${ }^{1, *}$, Jinquan $\mathrm{Ma}^{2}$ \\ ${ }^{1}$ Information Engineering University, The Information System Engineering Institute,, 450002 Zheng Zhou, China \\ ${ }^{2}$ Information Engineering University, The Information System Engineering Institute,, 450002 Zheng Zhou, China
}

\begin{abstract}
Based on MUSIC-LIKE algorithm, an improved algorithm for multiple source direction of arrival estimation was proposed to resolve the problem, the enabled estimation number of source for traditional direction of arrival estimation algorithm restricted by the size of the antenna array. The computations and the matrix dimension were reduced by constructed new fourth cumulate matrix, the number of direction estimation was increased by smoothing computation snapshots based on MFOCMUSIC algorithm. The spatial spectrum measuring error was decreased by smoothing the virtual array extended by fourth order cumulate. Simulation results proved that the proposed algorithm can find more direction of arrival than MFOC-MUSIC algorithm, improve the direction of arrival resolution ratio, and inhibit the influence of gaussian white noise to direction of arrival estimation
\end{abstract}

\section{Introduction}

As the estimation of direction of arrival (DOA) widely used on the radar, sonar, geological exploration and radio astronomy and other fields, higher requirement was proposed on the DOA algorithm. However, the number of spatial sources accurately estimated was restricted by the antenna array size. Since Dogan M C, Mendel J M [1]-[3] proposed the fourth order cumulate could bring about the virtual array units, more and more scholar started to discuss the way to expand the number of the virtual array to increase the accurately estimated spatial sources, such as Porat B and Friedlander B[4] put forward the MUSIC-LIKE algorithm and Shan Zhilong[5] found the effect of the rank of matrix on estimation direction. Based on the theory of virtual array expanded, Tang Jianhong[6] proposed the MFOCMUSIC algorithm to reduce the computational. However, most of the algorithm were restricted by the size of array size. In this paper, we reduced the error of measurement by smoothing the virtual array and increased the number of direction estimated by smoothing the consequence of snapshot spatial spectral function. The result of simulation indicates that the improved algorithm for multiple source direction of arrival estimation could estimate more direction than the MFOC-MUSIC algorithm and weaken the influence of the measurement error on estimation.

\section{Model and principle}

\subsection{Signal model}

We consider a uniform linear arrays(ULA) with $M$ sensor elements. The model is presented in figure1. The $N$ far failed signals were received by the antennas. Thus, consider $N$ number of non-Gaussian signals independent of the noise imping on a (ULA)from different directions $\theta$. And the antenna elements are separated from each other by a distance of $\mathrm{d}=\frac{\lambda}{2}$, where $\lambda$ is the wavelength of the wave impinging on the array. The antenna received matrix $\boldsymbol{X}(t)=\left[\begin{array}{llll}\boldsymbol{x}_{\boldsymbol{1}}(t) & \boldsymbol{x}_{2}(t) & \cdots & \boldsymbol{x}_{m}(t)\end{array}\right] \quad$ is indicated by $\boldsymbol{X}(t)=\boldsymbol{A}^{*} \boldsymbol{S}(t)+\boldsymbol{N}(t)$, where $\boldsymbol{S}(t)$ was indicated the signal source, the $N(t)$ indicated the noise vector, the steering vector of the antenna is given as $\boldsymbol{A}=\left[\boldsymbol{a}\left(\theta_{1}\right), \boldsymbol{a}\left(\theta_{2}\right), \cdots, \boldsymbol{a}\left(\theta_{N}\right)\right] \quad \boldsymbol{a}\left(\theta_{n}\right)(n=1,2 \cdots N) \in \mathbb{C}^{M^{* 1}}$, the $\boldsymbol{a}\left(\theta_{n}\right)$ is indicated as $\boldsymbol{a}\left(\theta_{n}\right)=\left[1, e^{j \omega \tau\left(\theta_{n}\right)} \cdots, e^{j \omega(m-1) \tau\left(\theta_{n}\right)}\right]^{T}$, the $\tau_{m}\left(\theta_{n}\right)$ is given as $\frac{d \cos \left(\theta_{n}\right)}{\lambda}$.

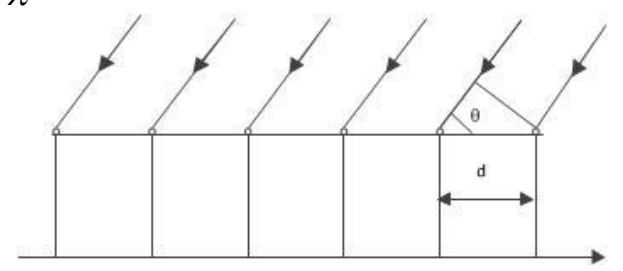

Fig. 1. Uniform Linear Array model.

\subsection{The algorithm of MUSIC-LIKE}

\footnotetext{
*Xuecheng Wang: iota9411@gmail.com
} 
It is an important feature that the fourth order cumulant could obtain the virtual array. Thus, the MUSIC-LIKE algorithm utilized the feature to expand the size of the antenna and increase the number of sensor elements to increase the number of the accurately estimation source. The fourth order cumulant of output matrix $\boldsymbol{X}(t)=\left[\begin{array}{llll}\boldsymbol{x}_{1}(t) & \boldsymbol{x}_{2}(t) & \cdots & \boldsymbol{x}_{m}(t)\end{array}\right]$ of MUSIC-LIKE algorithm was given as equation(1), the (E) denoted the mean value of the brackets, the $(*)$ denoted the matrix transpose, where the $(\mathrm{H})$ denoted the matrix conjugate transpose, the $(\otimes)$ denoted the Kronecker product.

$$
\begin{aligned}
& \boldsymbol{C}=\mathrm{E}\left\{\left(\boldsymbol{X} \otimes \boldsymbol{X}^{*}\right)\left(\boldsymbol{X} \otimes \boldsymbol{X}^{*}\right)^{\boldsymbol{H}}\right\} \\
& -\mathrm{E}\left\{\left(\boldsymbol{X} \otimes \boldsymbol{X}^{*}\right)\right\} \mathrm{E}\left\{\left(\boldsymbol{X} \otimes \boldsymbol{X}^{*}\right)^{\boldsymbol{H}}\right\} \\
& -\mathrm{E}\left\{\left(\boldsymbol{X} \boldsymbol{X}^{\boldsymbol{H}}\right)\right\} \otimes \mathrm{E}\left\{\left(\boldsymbol{X} \boldsymbol{X}^{\boldsymbol{H}}\right)^{*}\right\}
\end{aligned}
$$

As for ULA, the array guidance vector was given as form equation(2) to equation (5)

$$
\begin{gathered}
\boldsymbol{B}(\theta)=\left[\boldsymbol{b}\left(\theta_{1}\right), \boldsymbol{b}\left(\theta_{2}\right), \cdots, \boldsymbol{b}\left(\theta_{m^{2}}\right)\right] \\
\boldsymbol{b}(\theta)=\boldsymbol{a}(\theta) \otimes \boldsymbol{a}^{*}(\theta) \\
\boldsymbol{a}(\theta)=\left[1, e^{j \omega \tau(\theta)} \cdots, e^{j \omega(m-1) \tau(\theta)}\right]^{T} \\
\boldsymbol{b}(\theta)=\boldsymbol{a}(\theta) \otimes \boldsymbol{a}^{*}(\theta) \\
=\left[1, e^{j \omega \tau(\theta)} \cdots, e^{j \omega(m-1) \tau(\theta)}, e^{-j \omega \tau(\theta)},\right. \\
1, e^{j \omega \tau(\theta)} \cdots, e^{j \omega(m-2) \tau(\theta)}, e^{-j \omega 2 \tau(\theta)}, e^{-j \omega \tau(\theta)}, \\
1, \cdots]^{T}
\end{gathered}
$$

The MUSIC-LIKE algorithm need to decompose the matrix from the equation (1) to obtain the eigenvalues. Then, the algorithm took the eigenvector into the equation (6) to obtain the direction of the signal arrival.

$$
P(\theta)=\frac{1}{\left\{\boldsymbol{b}^{\boldsymbol{H}}(\theta) \boldsymbol{U}_{\text {noise }}\right\}^{2}}
$$

\subsection{The algorithm of MFOC-MUSIC}

Based on the Kronecker product, the dimensions of the MUSIC-LIKE matrix and the computation of the algorithm raise up. For example, the matrix $C \in \mathbb{C}^{M^{2 *} M^{2}}$, the vector $b(\theta) \in \mathbb{C}^{M^{2 * 1}}$, the dimensions larger than the classical MUSIC algorithm. The Structure of the $b(\theta)$ verified that greatly data of the vector was redundancy and replicate, similarly the matrix $C$. Thus, the scholar proposed the MFOC-MUSIC algorithm. According to the position of the virtual array in the $b(\theta)$, the algorithm proposed the new vector given as the equation(7). Based on the Kronecker product, retained the second order statistic information of the $\boldsymbol{X}(t)$. So that we could create the new fourth order cumulant matrix given as (8)

$$
\begin{aligned}
& b^{\prime}(\theta)=\left[1, e^{j \omega \tau(\theta)} \cdots, e^{j \omega(m-1) \tau(\theta)}, e^{-j \omega \tau(\theta)},\right. \\
& \left.e^{-j \omega 2 \tau(\theta)}, e^{-j \omega 3 \tau(\theta)}, \cdots, e^{-j \omega(m-1) \tau(\theta)}\right]^{T}
\end{aligned}
$$

$$
\begin{aligned}
& \boldsymbol{C}_{42_{(M+1)^{*}(\mathrm{M}+1)}}^{\prime}=\boldsymbol{C}_{\left.42_{(M+1)^{*}(M+1}\right)} \\
& \boldsymbol{C}_{42_{(1: \mathrm{M}+2)^{*}(\mathrm{M}+2)}^{\prime}}^{\prime}=\boldsymbol{C}_{42_{(1: \mathrm{M}+2)^{*}(2 M+1)}}=\boldsymbol{C}_{42_{(1: \mathrm{M}+3)^{*}(3 M+1)}} \\
& \boldsymbol{C}_{42_{(1: \mathrm{M}+3)^{*}(\mathrm{M}+3)}^{\prime}}^{\prime}=\boldsymbol{C}_{42_{(2 \mathrm{M}+1)^{*}(1: M+2)}} \\
& \boldsymbol{C}_{42_{(\mathrm{M}+2)^{*}(1: \mathrm{M}+2)}^{\prime}}^{\prime}=\boldsymbol{C}_{42_{(3 \mathrm{M}+)^{*}(1: M+3)}} \\
& \boldsymbol{C}_{42_{(\mathrm{M}+3)^{*}(1: \mathrm{M}+3)}^{\prime}}^{\prime}
\end{aligned}
$$

\section{The improved algorithm for multiple source direction of arrival estimation}

The MFOC-MUSIC algorithm reduced the fourth order cumulant computation and the dimension of the matrix, nevertheless, the algorithm could not increase the number of the estimation source. Based on the MFOCMUSIC algorithm, the paper proposed the improved algorithm for multiple source direction of arrival estimation. The algorithm expanded the number of the estimation source and reduced the measurement error by increased the number of snapshots and smoothed the consequence of the spatial spectrum function.

\subsection{The principle of the algorithm}

Compared the equation (5) with the equation(7), the paper proposed the new fourth order cumulant matrix $\boldsymbol{C}_{42_{(2 M-1)^{*}(2 \mathrm{M}-1)}}^{*}$, as the $\boldsymbol{b}(\theta)$ showed, the index of the first virtual array was $(k M+k)$, and the next index of the first virtual array was $(k M+k-1)$. The rest index could be done in the same manner, so that the mth index of the first virtual was $(k M+k-m+1)$, the paper smoothed the data of the same virtual array with different index as the new virtual array. When the $M=5$, the $a(\theta)$ was given as(9), the $b(\theta)$ was given as(10), the model was showed as figure 2,black circle depicted the real sensor elements, the polygon depicted the virtual sensor elements, the white circle depicted the ULA, respectively. The algorithm smoothed the data of the polygon column to the white circle sensor elements as the new sensor elements.

$$
\boldsymbol{a}(\theta)=\left[1, e^{j \omega \tau(\theta)}, e^{j \omega 2 \tau(\theta)}, e^{j \omega 3 \tau(\theta)}, e^{j \omega 4 \tau(\theta)}\right]^{T}
$$




$$
\begin{aligned}
& \boldsymbol{b}(\theta)=\boldsymbol{a}(\theta) \otimes \boldsymbol{a}^{*}(\theta) \\
& =\left[1, e^{j \omega \tau(\theta)}, e^{j \omega 2 \tau(\theta)}, e^{j \omega 3 \tau(\theta)}, e^{j \omega 4 \tau(\theta)},\right. \\
& e^{-j \omega \tau(\theta)}, 1, e^{j \omega \tau(\theta)}, e^{j \omega 2 \tau(\theta)}, e^{j \omega 3 \tau(\theta)}, \\
& e^{-j \omega 2 \tau(\theta)}, e^{-j \omega \tau(\theta)}, 1, e^{j \omega \tau(\theta)}, e^{j \omega 2 \tau(\theta)}, \\
& e^{-j \omega 3 \tau(\theta)}, e^{-j \omega 2 \tau(\theta)}, e^{-j \omega \tau(\theta)}, 1, e^{j \omega \tau(\theta)}, \\
& \left.e^{-j \omega 4 \tau(\theta)}, e^{-j \omega 3 \tau(\theta)}, e^{-j \omega 2 \tau(\theta)}, e^{-j \omega \tau(\theta)}, 1\right] \\
& \vdots \\
& \vdots \\
& \vdots \\
& + \\
& +
\end{aligned}
$$

Fig.2. $M=5$ Virtual array unit model

Smoothed the matrix $C_{42_{(2 M-1)^{*}(2 M-1)}^{\prime}}^{\prime}$ to get the new matrix $C_{42_{(2 M-1)^{*}(2 \mathrm{M}-1)}}^{*}$, the process was showed as equation.

$$
\begin{aligned}
& \boldsymbol{C}_{42_{(5) *(5)}^{*}}^{*}=\boldsymbol{C}_{42_{(5)^{*}(5)}}
\end{aligned}
$$

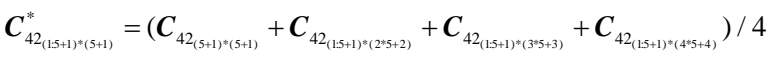

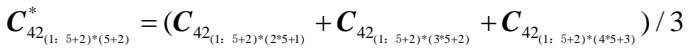

$$
\begin{aligned}
& \boldsymbol{C}_{42_{(1: 5+3)}^{*} *(5+3)}^{*}=\left(\boldsymbol{C}_{42_{(1: 5+3) *(355+1)}}+\boldsymbol{C}_{42_{(1.553) *(45+2)}}\right) / 2 \\
& \boldsymbol{C}_{42_{(1.5+4)^{*}(5+4)}^{*}}^{*}=\boldsymbol{C}_{42_{(1: 5+4) *(45+1)}}
\end{aligned}
$$

When we got the matrix $C_{42_{(2 M-1)^{*}(2 M-1)}^{*}}^{*}$, we got the eigenvector of the matrix, respectively as signal subspace eigenvectors and noise subspace eigenvectors. Then took the eigenvector into equation (6) to get the spatial spectrum value. Afterwards, we smoothed the different spatial spectrum value as the new spatial spectrum value. The direction of the spike value was the estimated signal directions.

\subsection{The flow of the algorithm}

(1) Got the $\boldsymbol{X}(t)$ from the real sensor elements and Kronecker product to get the fourth order cumulant matrix $\boldsymbol{C}_{42_{M^{2} M^{2}}}$;

(2) Smoothed the matrix $\boldsymbol{C}_{42_{M^{2} M^{2}}}$ as the equation (11) showed to get the new fourth order cumulant matrix $C_{42_{(2 M-1)^{*}(2 \mathrm{M}-1)}^{*}}^{*}$;

(3) Got the eigenvector of the matrix $C_{42}^{*}$

took the eigenvector into (6) to get the spatial spectrum value;

(4) Repeat the step1 to step3 for different snapshots, and smoothed the spatial spectrum value of different snapshots;

\section{Simulation}

In this section, the performance of the proposed algorithm and the MFOC-MUSIC algorithm was investigated and compared with the performance of the number of accurately estimation directions.

\subsection{Test 1}

We consider $N=9$ imping on an array of $M=4$ antenna elements from direction $-40^{\circ},-30^{\circ},-20^{\circ}$,$10^{\circ}, 0^{\circ}, 10^{\circ}, 20^{\circ}, 30^{\circ}, 40^{\circ}$ Each signal vector is considered to be independent from the source vectors at another time instances and to have the zero mean Gaussian white noise, $\mathrm{SNR}=10 \mathrm{~dB}$, got 512 spots for every snapshot, and consider smoothed 10 snapshots as an new snapshots data. Compared the algorithm with MFOC-MUSIC algorithm on the term of the estimation number of the direction, the simulation result was presented at figure 3 .

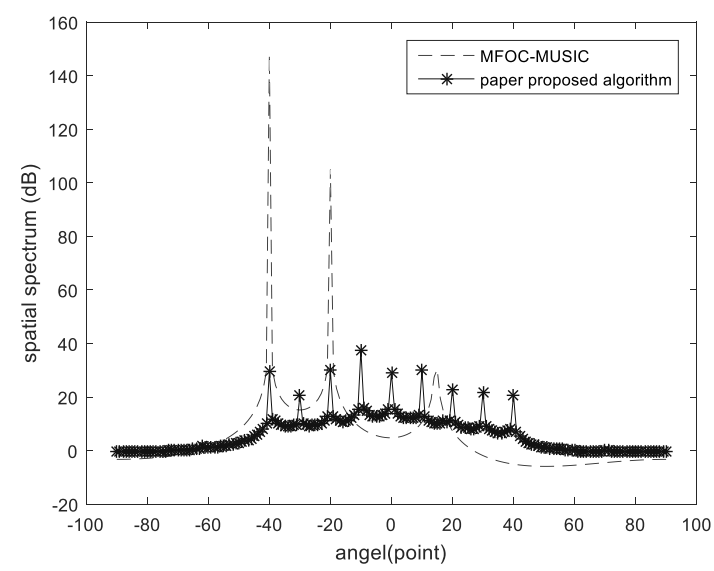

Fig. 3. Compared the MFOC-MUSIC algorithm with the improved algorithm for multiple source direction of arrival estimation

As the result depicted the improved algorithm for multiple source direction of arrival estimation could estimate more number of the signal direction than the MFOC-MUSIC algorithm. So that, the paper proposed algorithm can increase the number of estimation direction based on the limit ULA.

\subsection{Test 2}

In order to explore the influence of the volume of data on the experiment, we add the spots from the 512 for every snapshot to 2048 for every snapshot, and we took the same experiment as test 1 . The result was presented on figure4. 


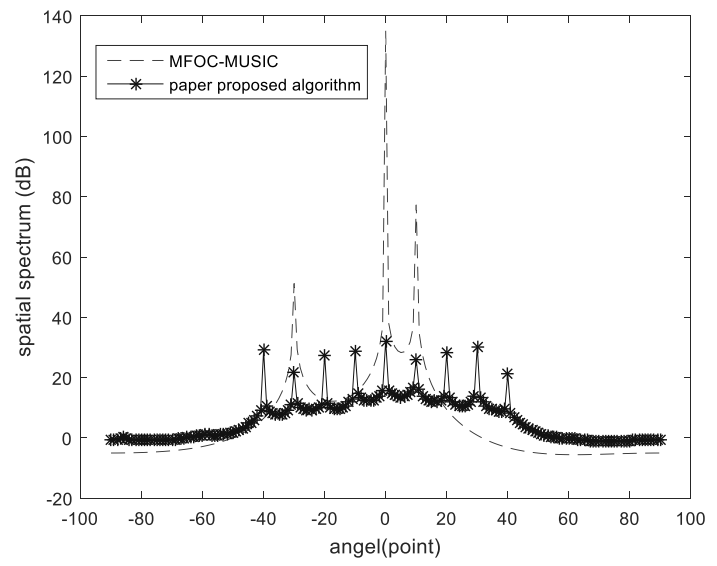

Fig.4. spot $=2048$ compared the MFOC-MUSIC algorithm with the improved algorithm for multiple source direction of arrival estimation

Based on the condition of testl experiment, we add the spots of the MFOC-MUSIC algorithm for every snapshot from 512 to 2048, and the spots of the paper proposed remain unchanged. The simulation result is presented as figure5.

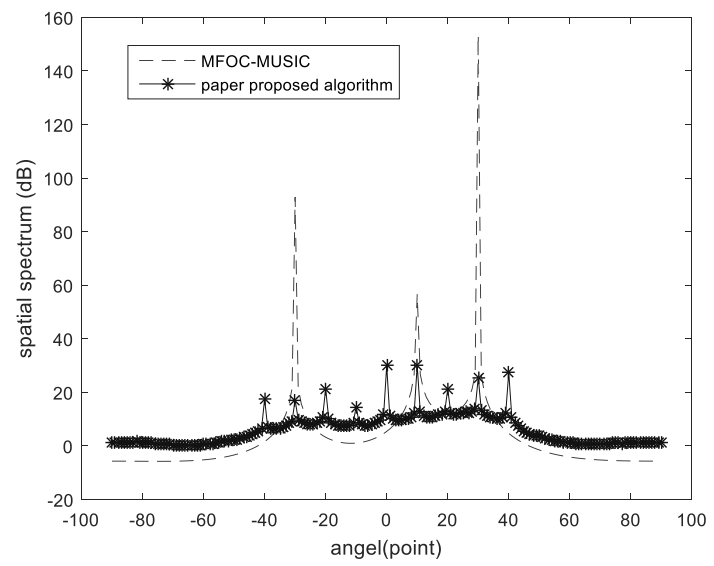

Fig.5. spot $_{M F O C-M U S I C}=2048$ spot $_{\text {MUSIC-LIKE }}=512$

Compared the MFOC-MUSIC algorithm the improved algorithm for multiple source direction of arrival estimation

As seen from figure4 and figure5, it is depicted that the number of the spots for every snapshot could not increase the number of estimation direction.

\section{Conclusion}

As simulation result showed, the improved algorithm for multiple source direction of arrival estimation greatly increased the number of estimation direction by smoothed the spatial spectrum value of the different snapshots compared with MFOC-MUSIC algorithm. The algorithm which proposed by the paper reduced the computation at the same time by deleting the repeated data of the matrix. And the algorithm reduced the measurement error by smoothed the data from the same virtual array.

\section{References}

1. Dogan M C, Mendel J M. Signal Processing IEEE Transactions on, 43(5):1200-1216. (2002)

2. Dogan M C, Mendel J M. IEEE Transactions on Signal Processing, 43(7):1663-1676.( 1995)

3. Porat B, Friedlander B. IEEE Transactions on Signal Processing, 39(9):2016-2024.( 1991)

4. Shan Z L, Liu X B, Wei Gang. Institute of Communication, 25(11):151-157. (2004)

5. Tang J H, Si X C, Chu P. System Engineering and Electronics. 32(2):256-259. (2010)

6. Zhang X F, Wang F, Xu D Z. Beijing: National Defense Industry Press.,11. (2010)

7. Wu S J, Zhang J Z, Zhang S. Journal of Harbin Engineering university.,26(03):394-397. (2005)

8. Wang Y L, Peng Y N, Chen Hui. Qin Hua University Press. (2004)

9. Shaghaghi M, Vorobyov S A. IEEE Transactions on Signal Processing, 63(12):3251-3265.( 2015)

10. Yan F G, Yan X W, Shi J, et al. Signal Processing, 139: 156-164.( 2017)

11. Liu L, Liu H. IEEE Access, 4:3815-3823.( 2017)

12. Liu Q H, Zhou X Q, Jin L N. Acta Aeronautica et Astronautican Sinica, 38(4):207-216. (2017)

13. Cai J J, Zong R, Cai H. Jounal of electronics and information technology, 38(1):168-173. (2016)

14. Liu Y, Buck J R. Asilomar Conference on. IEEE, 2017:944-948.( 2016)

15. Shi Y W, Chen M, Shan Z T, et al. Jilin University study paper, 47(1):268-273.( 2017) 